\title{
Bordetella parapertussis
}

National Cancer Institute

\section{Source}

National Cancer Institute. Bordetella parapertussis. NCI Thesaurus. Code C86205.

A species of aerobic, Gram-negative, coccobacilli shaped bacteria assigned to the phylum Proteobacteria. This species is nonmotile, oxidase negative, urease and citrate positive, does not reduce nitrate, and grows on MacConkey agar. B. parapertussis is found in the respiratory tract of mammals where it usually causes a mild form of whooping cough. 